\title{
Pembrolizumab for Treatment-Refractory Metastatic Castration-Resistant Prostate Cancer: Multicohort, Open-Label Phase II KEYNOTE-199 Study
}

Emmanuel S. Antonarakis, MD; Josep M. Piulats, MD, PhD²; Marine Gross-Goupil, MD, PhD³ Jeffrey Goh, MBBS ${ }^{4,5}$ Kristiina Ojamaa, MD ${ }^{6}$; Christopher J. Hoimes, DO; Ulka Vaishampayan, MD ${ }^{8}$; Ranaan Berger, MD, PhD ${ }^{9}$; Ahmet Sezer, MD ${ }^{10}$; Tuomo Alanko, MD, PhD ${ }^{11}$; Ronald de Wit, MD, PhD ${ }^{12}$; Chunde Li, MD, PhD ${ }^{13}$; Aurelius Omlin, MD ${ }^{14}$; Giuseppe Procopio, MD ${ }^{15}$; Satoshi Fukasawa, MD ${ }^{16}$; Ken-ichi Tabata, MD, PhD ${ }^{17}$; Se Hoon Park, MD ${ }^{18}$; Susan Feyerabend, MD ${ }^{19}$; Charles G. Drake, MD, PhD ${ }^{20}$; Haiyan Wu, PhD ${ }^{21}$; Ping Qiu, PhD ${ }^{22}$; Jeri Kim, MD ${ }^{22}$; Christian Poehlein, MD ${ }^{22}$; and Johann Sebastian de Bono, MD, PhD ${ }^{23}$

PURPOSE Pembrolizumab has previously shown antitumor activity against programmed death ligand 1 (PD-L1)positive metastatic castration-resistant prostate cancer (mCRPC). Here, we assessed the antitumor activity and safety of pembrolizumab in three parallel cohorts of a larger mCRPC population.

METHODS The phase II KEYNOTE-199 study included three cohorts of patients with mCRPC treated with docetaxel and one or more targeted endocrine therapies. Cohorts 1 and 2 enrolled patients with RECISTmeasurable PD-L1-positive and PD-L1-negative disease, respectively. Cohort 3 enrolled patients with bonepredominant disease, regardless of PD-L1 expression. All patients received pembrolizumab $200 \mathrm{mg}$ every 3 weeks for up to 35 cycles. The primary end point was objective response rate per RECIST v1.1 assessed by central review in cohorts 1 and 2. Secondary end points included disease control rate, duration of response, overall survival (OS), and safety.

RESULTS Two hundred fifty-eight patients were enrolled: 133 in cohort 1, 66 in cohort 2, and 59 in cohort 3. Objective response rate was $5 \%(95 \% \mathrm{Cl}, 2 \%$ to $11 \%)$ in cohort 1 and $3 \%(95 \% \mathrm{Cl},<1 \%$ to $11 \%)$ in cohort 2 . Median duration of response was not reached (range, 1.9 to $\geq 21.8$ months) and 10.6 months (range, 4.4 to 16.8 months), respectively. Disease control rate was $10 \%$ in cohort $1,9 \%$ in cohort 2, and $22 \%$ in cohort 3 . Median OS was 9.5 months in cohort 1, 7.9 months in cohort 2, and 14.1 months in cohort 3. Treatment-related adverse events occurred in $60 \%$ of patients, were of grade 3 to 5 severity in $15 \%$, and led to discontinuation of treatment in $5 \%$.

CONCLUSION Pembrolizumab monotherapy shows antitumor activity with an acceptable safety profile in a subset of patients with RECIST-measurable and bone-predominant mCRPC previously treated with docetaxel and

ASSOCIATED CONTENT

See accompanying Editorial on page $\mathbf{3 8 1}$ Appendix

Data Supplement Author affiliations and support information (if applicable) appear at the end of this article.

Accepted on September 30, 2019 and published at ascopubs.org/journal/ jco on November 27, 2019: Dol https://doi. org/10.1200/JCO.19. 01638 targeted endocrine therapy. Observed responses seem to be durable, and OS estimates are encouraging.

J Clin Oncol 38:395-405. @ 2019 by American Society of Clinical Oncology

\section{INTRODUCTION}

In the past decade, therapeutic options for advanced prostate cancer have increased secondary to improved understanding of the molecular mechanisms that underlie metastatic progression, including the critical role of the tumor microenvironment. ${ }^{1}$ Metastatic prostate cancer initially responds to androgen deprivation, the long-standing standard of care. More recent trials have shown that adding docetaxe ${ }^{2-4}$ or abiraterone acetate ${ }^{5,6}$ to androgen deprivation improves overall survival (OS) in patients with metastatic hormone-sensitive disease. Eventually, tumors stop responding to androgen deprivation, a state referred to as castrate-resistant prostate cancer (CRPC). ${ }^{7}$ For patients with metastatic CRPC (mCRPC), treatment options that confer a survival benefit include docetaxel, $, 8,9$ cabazitaxel, ${ }^{10}$ abiraterone, ${ }^{11,12}$ enzalutamide, ${ }^{13,14}$ sipuleucel-T, 15 and the bone-specific radionuclide radium-223. ${ }^{16}$ These therapies are not curative and may be associated with poor tolerability.

Monoclonal antibodies that target cytotoxic T-lymphocyteassociated protein 4, programmed death 1 receptor (PD-1), and programmed death ligand 1 (PD-L1) have demonstrated antitumor activity and manageable safety in several advanced malignancies. Although checkpoint inhibition has demonstrated efficacy in urothelial and renal-cell carcinomas, ${ }^{17-25}$ prostate cancer has a more immunosuppressive microenvironment than these 
other genitourinary malignancies, ${ }^{26-28}$ which suggests that mCRPC may be less susceptible to immune checkpoint blockade. The cytotoxic T-lymphocyte-associated protein 4 inhibitor ipilimumab failed to significantly prolong OS in patients with mCRPC that progressed on docetaxe ${ }^{29}$ or was chemotherapy naive. ${ }^{30}$ Recently, the humanized, anti-PD-1 monoclonal antibody pembrolizumab has demonstrated antitumor activity and manageable safety in patients with mCRPC. In 23 patients with PD-L1-positive MCRPC who were enrolled in KEYNOTE-028, three quarters of whom had received two or more lines of previous therapy, pembrolizumab provided a $17 \%$ objective response rate (ORR), a $30 \%$ disease control rate (DCR), and a $37 \%$ estimated 12 -month OS rate. ${ }^{31}$ Initial results from the first 10 patients with enzalutamide-resistant MCRPC who were treated with pembrolizumab in a phase II study showed a rapid decrease in prostate-specific antigen (PSA) levels for three patients, radiographic partial response in two patients, and radiographic stable disease in three patients. ${ }^{32}$

To further explore the antitumor activity and safety of pembrolizumab in mCRPC, we performed the KEYNOTE199 study. We report results for the first three cohorts, which enrolled in parallel and included patients who previously received docetaxel and targeted endocrine therapy for disease that was measurable and PD-L1 positive (cohort 1 ) or negative (cohort 2) or that was bone predominant, regardless of PD-L1 status (cohort 3 ).

\section{METHODS}

\section{Study Design and Patients}

KEYNOTE-199 is a five-cohort, open-label, phase II study. Cohorts 1, 2, and 3 enrolled patients at 85 sites in 21 countries. The trial was conducted in accordance with Good Clinical Practice and the protocol and its amendments, which were approved by the appropriate ethics body at each center. All patients provided written informed consent.

Key eligibility criteria for cohorts 1 to 3 included age 18 years or older; metastatic or locally confined but inoperable, pathologically confirmed prostate adenocarcinoma; measurable disease per RECIST $v 1.1^{33}$ (cohorts 1 and 2) or detectable bone metastases by whole-body bone scintigraphy and no RECIST-measurable tumors (cohort 3) by central review; Eastern Cooperative Oncology Group performance status 0,1 , or 2; provision of a tumor sample for PD-L1 assessment (cohort 1 limited to PD-L1-positive disease, cohort 2 limited to PD-L1-negative disease); and previous treatment with one or more targeted endocrine therapies and one to two chemotherapy regimens, one of which must have included docetaxel. Full inclusion and exclusion criteria are in the protocol (available in the Data Supplement).

\section{Treatment}

Pembrolizumab 200 mg was administered intravenously every 3 weeks until disease progression, intolerable toxicity, physician decision, withdrawal of consent, or a maximum of 35 cycles was reached. Patients with confirmed complete response who received eight or more pembrolizumab cycles, including two or more cycles beyond initial complete response, were permitted to discontinue treatment. Complete discontinuation criteria and adverse event (AE) management guidelines are available in the protocol.

\section{Assessments and End Points}

PD-L1 expression was assessed at a central laboratory in formalin-fixed, paraffin-embedded tumor samples using the PD-L1 IHC 22C3 pharmDx assay (Agilent Technologies, Carpinteria, CA). Patients in cohorts 1 and 2 were required to provide biopsy samples from metastatic tumor collected within 12 months of screening or an archival tumor specimen if collection from a metastasis was not feasible. Patients in cohort 3 were required to provide at least an archival specimen. PD-L1 positivity was defined as a combined positive score (CPS) of $\geq 1$, where CPS is the number of PD-L1-positive cells-tumor cells, lymphocytes, and macrophages - divided by the total number of tumor cells $\times$ 100. Mutations in DNA damage repair (DDR) genes were interrogated using whole-exome sequencing of DNA isolated from formalin-fixed, paraffin-embedded tumor samples. Complete details are found in the Data Supplement.

Computed tomography, radionuclide bone scanning, and PSA assessment were performed at baseline, every 9 weeks during year 1 , and every 12 weeks thereafter. AEs were collected throughout treatment and for 30 days thereafter90 days for serious AEs - and graded according to the National Cancer Institute Common Terminology Criteria for Adverse Events, version 4.0. Survival was assessed every 12 weeks during follow-up.

The primary end point was ORR per RECIST v1.1, assessed by central review in patients with measurable disease at baseline in cohorts 1 and 2 separately and combined. Secondary end points included DCR per RECIST v1.1, assessed by central review; ORR, DCR, and radiographic progression-free survival (rPFS) per Prostate Cancer Working Group 3 (PCWG3) -modified RECIST $\mathrm{v} 1.1,{ }^{34}$ assessed by central review; PSA response rate, and OS in cohorts 1 and 2 combined, cohorts 1, 2, and 3 combined, and each cohort separately; duration of response per RECIST V1.1 and per PCWG3-modified RECIST V1.1, assessed by central review for cohorts 1 and 2 combined and separately; and safety and tolerability. Detailed end point definitions are provided in the Data Supplement.

\section{Statistical Considerations}

The statistical analysis plan is available in the protocol in the Data Supplement. Antitumor activity and safety were analyzed in all patients who received one or more pembrolizumab doses. Patients without a baseline PSA level were excluded from PSA response analysis. ORR, DCR, and PSA response rate point estimates and $95 \% \mathrm{Cls}$ were 
calculated using the Clopper-Pearson exact binomial method. rPFS, OS, and response duration were estimated using the Kaplan-Meier method; censoring rules for time-to-event end points are summarized in the Data Supplement. No hypothesis testing was planned, and no multiplicity adjustments to control for Type I error were performed. The planned sample size was approximately 200 patients in cohorts 1 and 2 combined and 50 patients in cohort 3 and was designed to provide a $95 \% \mathrm{Cl}$ for ORR with an approximate width of $10 \%$.

\section{RESULTS}

\section{Patients}

Between July 1, 2016, and March 31, 2017, 260 of 394 patients who were screened for cohorts 1,2 , and 3 were enrolled (Fig 1). Cohort 1 included 133 patients with RECIST-measurable, PD-L1-positive disease. Cohort 2 included 65 patients with RECIST-measurable, PD-L1-negative disease and three patients with RECIST-measurable disease of unknown PD-L1 status. Cohort 3 included 59 patients with bone-predominant disease. Biopsy samples that were adequate for PD-L1 testing were acquired within 12 months of screening for 122 (92\%) of 133 patients in cohort 1 and 52 (79\%) of 66 in cohort 2 . These newly collected samples were from metastases in $98(80 \%)$ of 122 and 48 (92\%) of 52 patients, respectively. In cohort 3 , two $(9 \%)$ of 23 PD-L1-positive and five $(14 \%)$ of 36 PD-L1-negative samples were acquired within 12 months of screening; only one newly collected sample, which was PD-L1 negative, was from a metastasis.

Baseline characteristics were generally as expected for a docetaxel-treated mCRPC population (Table 1). Across cohorts, median age was 68 years, 36\% of patients had
Eastern Cooperative Oncology Group performance status $0,29 \%$ received two or more previous chemotherapy regimens, and $25 \%$ received two or more previous antiandrogen therapies. Median PSA level in cohorts 1,2, and 3 was $115.5 \mathrm{ng} / \mathrm{mL}, 116.1 \mathrm{ng} / \mathrm{mL}$, and $43.3 \mathrm{ng} / \mathrm{mL}$, respectively.

As of the August 21, 2018, data cutoff, median follow-up was 9.5 months in cohort 1, 7.9 months in cohort 2, and 14.1 months in cohort 3, and $4 \%, 0 \%$, and $3 \%$ of patients, respectively, remained on pembrolizumab (Fig 1 ). All but two patients in cohort 2 received one or more pembrolizumab doses. The most common reasons for treatment discontinuation were disease progression and AEs. Median treatment duration was 2.1 months (range, 0.03 to 21.4 months) in cohort $1,1.6$ months (range, 0.03 to 18.7 months) in cohort 2, and 3.2 months (range, 0.7 to 21.9 months) in cohort 3.

\section{Antitumor Activity}

Among the 199 patients in cohorts 1 and 2, 29\% had a decrease from baseline in the sum of target lesions, including $9 \%$ who had a $30 \%$ or greater decrease; $26 \%$ of patients had no change or a less than $20 \%$ increase in the sum of target lesions, with similar results in each cohort (Fig 2A). Considering only the 166 patients with one or more evaluable postbaseline imaging assessment, 34\% had any decrease, $10 \%$ had a $30 \%$ or greater decrease, and $31 \%$ had no change or a less than $20 \%$ increase in the sum of target lesions. ORR was $5 \%(95 \% \mathrm{Cl}, 2 \%$ to $11 \%)$ in cohort $1,3 \%(95 \% \mathrm{Cl},<1 \%$ to $11 \%)$ in cohort 2 , and $5 \%$ (95\% $\mathrm{Cl}, 2 \%$ to $8 \%$ ) in cohorts 1 and 2 combined, per both RECIST v1.1 and PCWG3-modified RECIST (Table 2). Two patients, both in cohort 1 , achieved a complete radiographic response. The remaining five responses in cohort 1

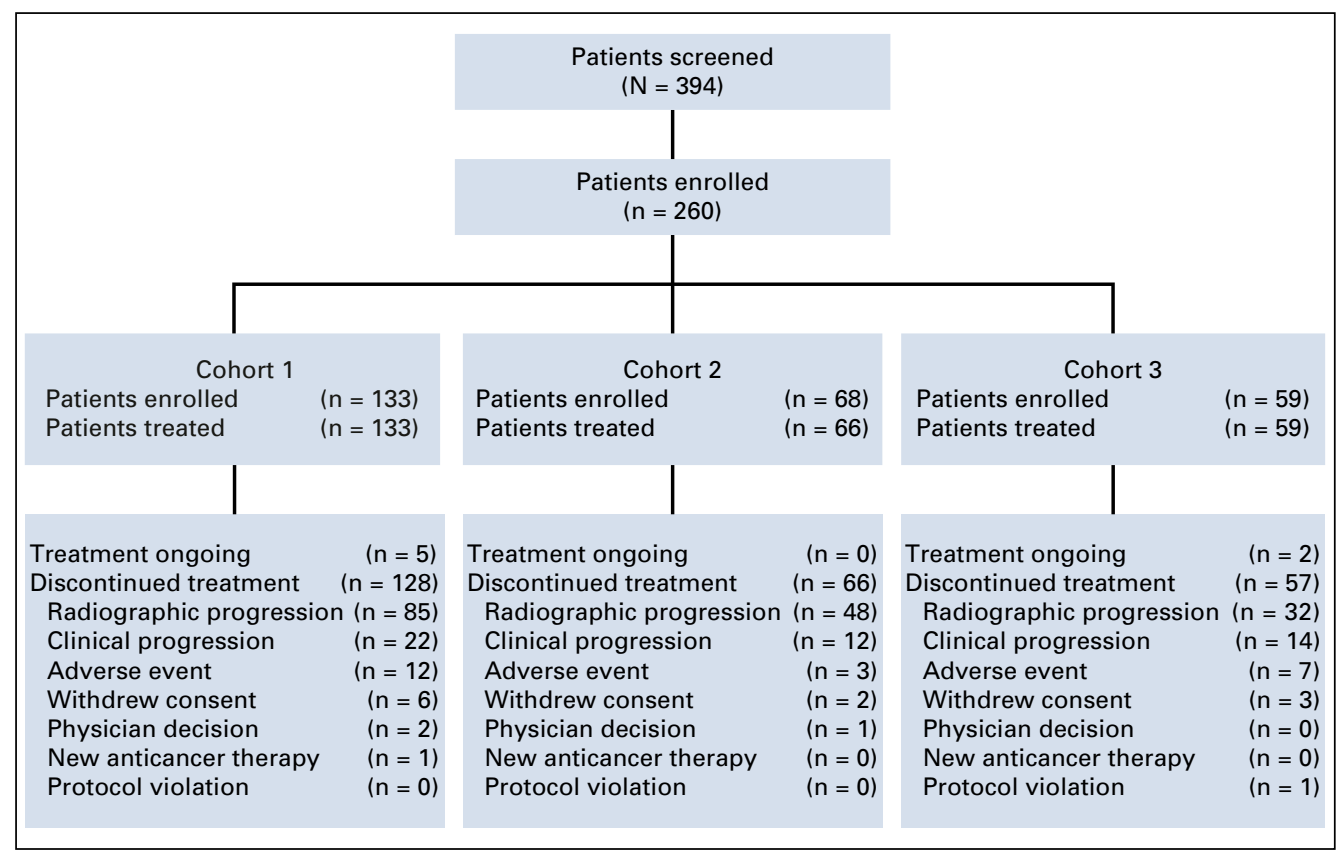

FIG 1. Patient disposition. 
TABLE 1. Patient Characteristics at Baseline

\begin{tabular}{|c|c|c|c|}
\hline \multirow[b]{3}{*}{ Characteristic } & \multirow{2}{*}{$\begin{array}{c}\text { Cohort } 1 \\
\text { PD-L1 Positive } \\
\end{array}$} & \multirow{2}{*}{$\begin{array}{c}\text { Cohort } 2 \\
\text { PD-L1 Negative } \\
\end{array}$} & \multirow{2}{*}{$\begin{array}{c}\text { Cohort } 3 \\
\text { Bone Predominant }\end{array}$} \\
\hline & & & \\
\hline & $(n=133)$ & $(n=66)$ & $(n=59)$ \\
\hline Median age, years (range) & $68(48-85)$ & $68(53-84)$ & $71(53-90)$ \\
\hline \multicolumn{4}{|l|}{ ECOG performance status } \\
\hline 0 & $42(32)$ & $25(38)$ & $26(44)$ \\
\hline 1 & $75(56)$ & $36(55)$ & $27(46)$ \\
\hline 2 & $16(12)$ & $4(6)$ & $6(10)$ \\
\hline Missing & 0 & $1(2)$ & 0 \\
\hline \multicolumn{4}{|l|}{ Gleason score } \\
\hline$\leq 7$ & $40(31)$ & $18(27)$ & $24(41)$ \\
\hline$\geq 8$ & $85(64)$ & $43(65)$ & $32(54)$ \\
\hline Unknown & $8(6)$ & $5(8)$ & $3(5)$ \\
\hline Median PSA value, ng/mL (range) & $115.5(0.1-5,000.0)$ & $116.1(1.0-3,583.0)$ & $43.3(0.1-2,539.0)$ \\
\hline Presence of visceral disease & $89(67)$ & $30(45)$ & $6(10)$ \\
\hline \multicolumn{4}{|c|}{ No. of previous chemotherapy regimens } \\
\hline 1 & $89(67)$ & $48(73)$ & $46(78)$ \\
\hline$\geq 2$ & $44(33)$ & $18(27)$ & $13(22)$ \\
\hline \multicolumn{4}{|c|}{ Types of previous targeted endocrine therapy } \\
\hline Enzalutamide only & $41(31)$ & $27(41)$ & $16(27)$ \\
\hline Abiraterone only & $57(43)$ & $24(36)$ & $28(47)$ \\
\hline Enzalutamide and abiraterone & $34(26)$ & $15(23)$ & $15(25)$ \\
\hline Other & $1(<1)$ & 0 & 0 \\
\hline
\end{tabular}

NOTE. Data are presented as No. (\%) unless otherwise indicated.

Abbreviations: ECOG, Eastern Cooperative Oncology Group; PD-L1, programmed death-ligand 1; PSA, prostate-specific antigen.

and both responses in cohort 2 were partial responses (Appendix Table A1, online only). Per RECIST v1.1, an additional six patients in cohort 1 and four patients in cohort 2 had stable disease for 6 months or longer leading to a DCR of $10 \%$ and $9 \%$, respectively (Table 2 ). DCR per PCWG3-modified RECIST was $13 \%$ in cohort $1,18 \%$ in cohort 2, 39\% in cohort 3,15\% in cohorts 1 and 2 combined, and $20 \%$ in cohorts 1,2 , and 3 combined (Table 2). Among the nine patients with radiographic response, four remained on treatment at data cutoff, three had experienced subsequent disease progression, and one had died without disease progression (Fig 3A). Median response duration was not reached (range, 1.9 to $\geq 21.8$ months) in cohort 1, was 10.6 months (range, 4.4 to 16.8 months) in cohort 2, and was 16.8 months (range, 1.9 to $\geq 21.8$ months) in cohorts 1 and 2 combined per both RECIST V1.1 and PCWG3-modified RECIST.

Median rPFS per PCWG3-modified RECIST was 2.1 months (95\% Cl, 2.0 to 2.1 months) in cohort 1, 2.1 months $(95 \% \mathrm{Cl}$, 2.0 to 3.3 months) in cohort 2, and 3.7 months ( $95 \% \mathrm{Cl}, 2.1$ to 4.2 months) in cohort 3 (Fig 3B). Median OS was 9.5 months $(95 \% \mathrm{Cl}, 6.4$ to 11.9 months) in cohort 1 ,
7.9 months ( $95 \% \mathrm{Cl}, 5.9$ to 10.2 months) in cohort 2, and 14.1 months ( $95 \% \mathrm{Cl}, 10.8$ to 17.6 months) in cohort 3 (Fig 3C). Estimated 12-month survival rates were $41 \%, 35 \%$, and $62 \%$, respectively. In cohorts 1 and 2 combined, median rPFS was 2.1 months (95\% Cl, 2.0 to 2.1 months) and median OS was 8.1 months (95\% Cl, 6.6 to 10.7 months). Median rPFS and OS were 2.1 months $(95 \% \mathrm{Cl}, 2.1$ to 2.2 months) and 9.6 months (95\% Cl, 7.9 to 12.2 months), respectively, in cohorts 1,2 , and 3 combined.

Of 243 patients in cohorts 1, 2, and 3 with baseline PSA assessment, 15\% experienced some decrease in PSA level, including $9 \%$ who had a $50 \%$ or greater decrease and $5 \%$ who had a $90 \%$ or greater decrease (Fig 2B). Stable PSA levels were observed in $9 \%$ of patients. Confirmed PSA responses occurred in $6 \%$ of 124 patients in cohort 1,8\% of 60 in cohort $2,2 \%$ of 59 in cohort $3,7 \%$ of 184 in cohorts 1 and 2 combined, and $6 \%$ of 243 in cohorts 1, 2, and 3 combined (Table 2).

\section{Exploratory Biomarker Analysis}

Across cohorts, 153 of 258 patients, including six of nine with complete or partial response, had tumor samples for 


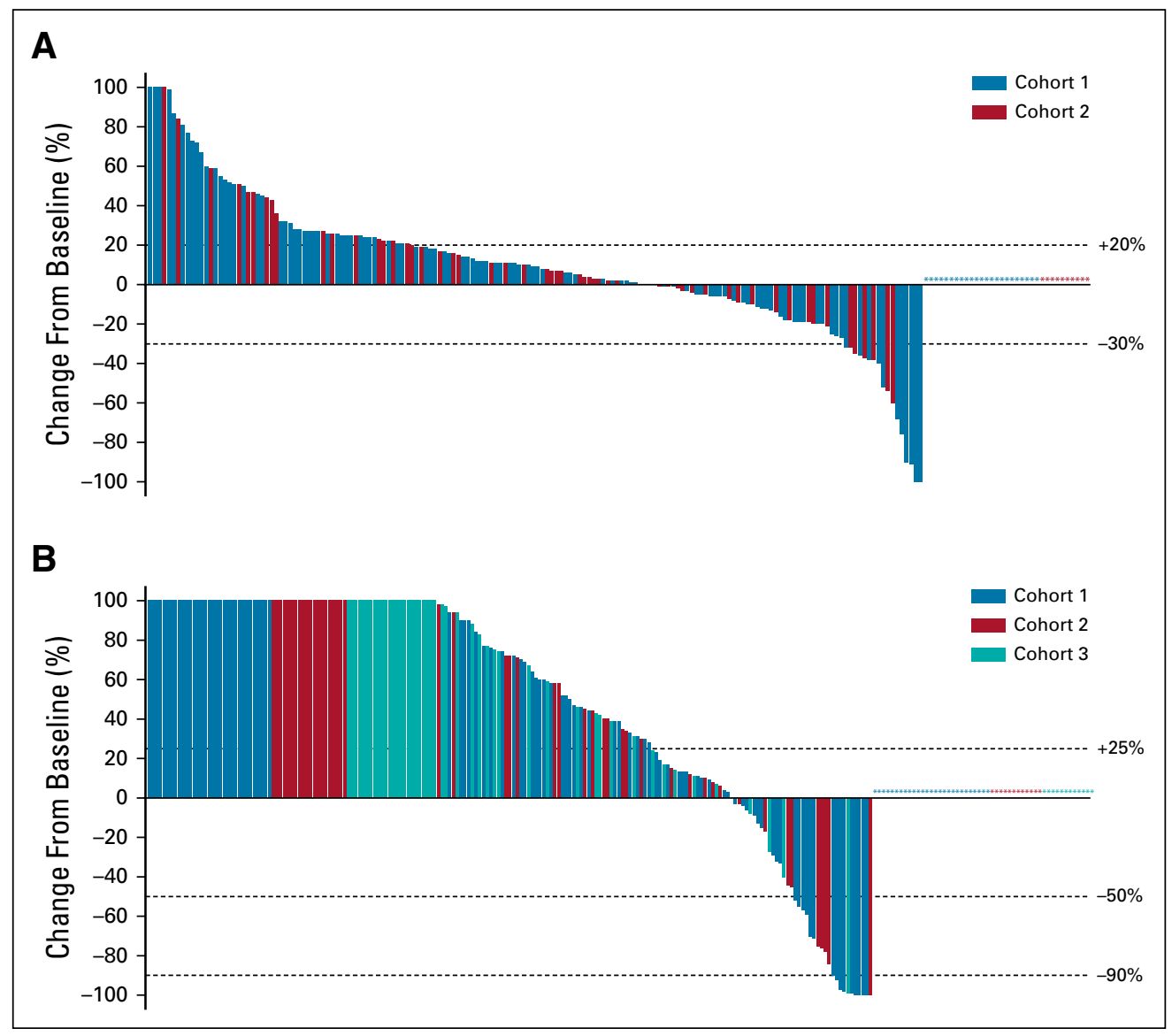

FIG 2. Waterfall plots of change from baseline in tumor size and prostate-specific antigen (PSA) level. (A) Best percentage change from baseline in the sum of longest diameters of target lesions as assessed by RECIST v1.1 by central review in the 199 patients enrolled in cohorts 1 and 2. Asterisks represent the 23 patients in cohort 1 and 10 patients in cohort 2 who were not evaluable for change from baseline in tumor size because they did not have one or more evaluable postbaseline imaging assessment. (B) Best percentage change from baseline in PSA level in the 243 patients enrolled in cohorts 1, 2, and 3 who had a baseline PSA measurement. Asterisks represent the 27 patients in cohort 1, 12 patients in cohort 2, and 11 patients in cohort 3 who had a baseline PSA measurement but did not have one or more postbaseline PSA measurement. Changes $>+100 \%$ were truncated at $+100 \%$.

which whole-exome sequencing was completed. Of these, $19(12 \%)$ had aberrations in BRCA1/2 or ATM and $10(7 \%)$ had aberrations in one or more of 12 other homologous recombination repair (HRR) genes (overall prevalence, 19\%; Appendix Table A2, online only). None of the six patients who experienced a response and had evaluable genomic data were determined to have microsatellite instability as defined by the mSINGS assay. ${ }^{35}$ ORR per RECIST v1.1 by central review was $11 \%$ in patients with BRCA1/2 or ATM aberrations, $0 \%$ in patients with aberrations in one or more of the 12 other HRR genes, and 3\% in patients without any HRR gene aberrations (Appendix Table A2). Response duration was 4.4 months in the patient with an ATM mutation and $\geq 21.8$ months in the patient with a BRCA2 mutation. Among the four responders without aberrations in HRR genes, response duration ranged from 1.9 months to $\geq 16.6$ months. Dedicated examination of 50 DDR genes in the six evaluable objective responders revealed that four patients had somatic aberrations in one or more of the genes evaluated (Appendix Table A3, online only). Local biomarker testing of two responding patients whose responses lasted more than 2 years revealed a mismatch repair defect by immunohistochemistry that was below the cutoff for high microsatellite instability by mSINGS with high tumor mutational burden and high CD3 intratumor infiltration in one patient and an amplified CD274 (PD-L1)/PDCD1LG2 (PD-L2) locus in the other.

\section{Safety}

Across cohorts, 155 (60\%) patients experienced one or more treatment-related AEs, including $39(15 \%)$ with one or more grade 3 to 5 treatment-related AEs and 12 (5\%) who discontinued pembrolizumab because of a treatmentrelated $A E$. Two patients died of $A E s$ that were considered to be treatment related by the investigator (pneumonitis and 
TABLE 2. Summary of Responses

\begin{tabular}{|c|c|c|c|c|c|}
\hline Variable & $\begin{array}{c}\text { Cohort } 1 \\
\text { (PD-L1 positive) }\end{array}$ & $\begin{array}{c}\text { Cohort } 2 \\
\text { (PD-L1 negative) }\end{array}$ & $\begin{array}{c}\text { Cohort } 3 \\
\text { (bone predominant) }\end{array}$ & $\begin{array}{c}\text { Cohorts } 1 \text { and } 2 \\
\text { Combined }\end{array}$ & $\begin{array}{c}\text { Cohorts } 1,2 \text {, and } 3 \\
\text { Combined }\end{array}$ \\
\hline \multicolumn{6}{|l|}{$\begin{array}{l}\text { Response assessed per RECIST } \\
\text { v1.1 by central radiology review }\end{array}$} \\
\hline No. of patients & 133 & 66 & 59 & 199 & 258 \\
\hline ORR, No. (\%; 95\% Cl) & $7(5 ; 2$ to 11$)$ & $2(3 ;<1$ to 11$)$ & - & $9(5 ; 2$ to 8$)$ & - \\
\hline DCR, ${ }^{*}$ No. $(\% ; 95 \% \mathrm{Cl})$ & $13(10 ; 5$ to 16$)$ & $6(9 ; 3$ to 19$)$ & $13(22 ; 12$ to 35$)$ & 19 (10; 6 to 15$)$ & $32(12 ; 9$ to 17$)$ \\
\hline No. of patients & 133 & 66 & 59 & 199 & 258 \\
\hline ORR, No. (\%; 95\% Cl) & $7(5 ; 2$ to 11$)$ & $2(3 ;<1$ to 11$)$ & - & $9(5 ; 2$ to 8$)$ & 一 \\
\hline DCR, ${ }^{*}$ No. $(\% ; 95 \%$ Cl) & $17(13 ; 8$ to 20$)$ & $12(18 ; 10$ to 30$)$ & $23(39 ; 27$ to 53$)$ & $29(15 ; 10$ to 20$)$ & $52(20 ; 15$ to 26$)$ \\
\hline Response rate, No. (\%; 95\% Cl) & $8(6 ; 3$ to 12$)$ & $5(8 ; 3$ to 18$)$ & $1(2 ; 0$ to 9$)$ & $13(7 ; 4$ to 12$)$ & $14(6 ; 3$ to 10$)$ \\
\hline
\end{tabular}

Abbreviations: DCR, disease control rate; ORR, objective response rate; PCWG3, Prostate Cancer Working Group 3; PD-L1, programmed death-ligand 1; PSA, prostate-specific antigen.

*Defined as the percentage of patients with confirmed complete or partial response of any duration or stable disease or noncomplete response or nonprogressive disease for 6 months or longer. Patients who died after month 6 without evidence of disease progression before death were considered to have stable disease for 6 months or longer.

†Defined as the percentage of patients with a reduction in PSA level from baseline by $50 \%$ or greater as confirmed on an additional PSA evaluation performed $\geq 3$ weeks later.

sepsis, $\mathrm{n}=1$ each). The most common treatment-related AEs were fatigue, diarrhea, and decreased appetite (Table 3). The only grade 3 to 5 treatment-related AEs with an incidence of $1 \%$ or greater were colitis and fatigue. Immune-mediated AEs and infusion reactions, which were based on a list of terms specified by the sponsor and considered regardless of attribution to treatment or immune relatedness by the investigator, occurred in 40 (16\%) patients, were grade 3 to 5 in 15 (6\%), and led to discontinuation in four (2\%) and death in one patient ( $<1 \%$; the aforementioned pneumonitis). The most common immune-mediated AEs were colitis, hyperthyroidism, hypothyroidism, pneumonitis, and severe skin reactions ( $n=6[2 \%]$ each; Table 3 ).

\section{DISCUSSION}

In this multicohort, open-label, phase II study, which to our knowledge is the largest trial of anti-PD-1 therapy in mCRPC conducted to date, we showed that pembrolizumab monotherapy has antitumor activity in patients with mCRPC previously treated with docetaxel and one or more targeted endocrine therapies, with approximately $25 \%$ of patients having received both enzalutamide and abiraterone. Observed OS estimates are promising, suggesting that immunotherapy may be able to extend the tail of the survival curve in a historically difficult-to-treat population. Pembrolizumab demonstrated activity in both
RECIST-measurable and bone-predominant disease, as well as in PD-L1-positive and PD-L1-negative tumors. Although there was only one PSA response in cohort 3, rPFS and OS were longer than in cohorts 1 and 2, reflecting different prognoses of bone-predominant and RECISTmeasurable disease. Pembrolizumab had manageable safety, and the overall toxicity profile was consistent with that previously observed for pembrolizumab.

Although the observed ORR was a modest 5\%, those responses that did occur were durable. Among the nine patients with RECIST-measurable disease who achieved complete or partial response, five had response ongoing at data cutoff, and median duration was 16.8 months. The prolonged response durability is consistent with what has been previously observed for pembrolizumab in other tumor types. ${ }^{17,36-39}$ To capture antitumor activity in bone metastases, which occur in $60 \%$ to $70 \%$ of men with advanced prostate cancer, ${ }^{40,41}$ this study also applied PCWG3modified response assessment. ${ }^{34}$ Whereas ORR and response duration in cohorts 1 and 2 were similar regardless of the criteria applied, DCR was higher in all cohorts using PCWG3-modified RECIST. These findings highlight the importance of accurately evaluating progression in bone metastases and suggest that PCWG3-modified RECIST may be preferable for assessing antitumor activity in MCRPC.

This study showed a clear signal of antitumor activity for pembrolizumab monotherapy in a small number of 


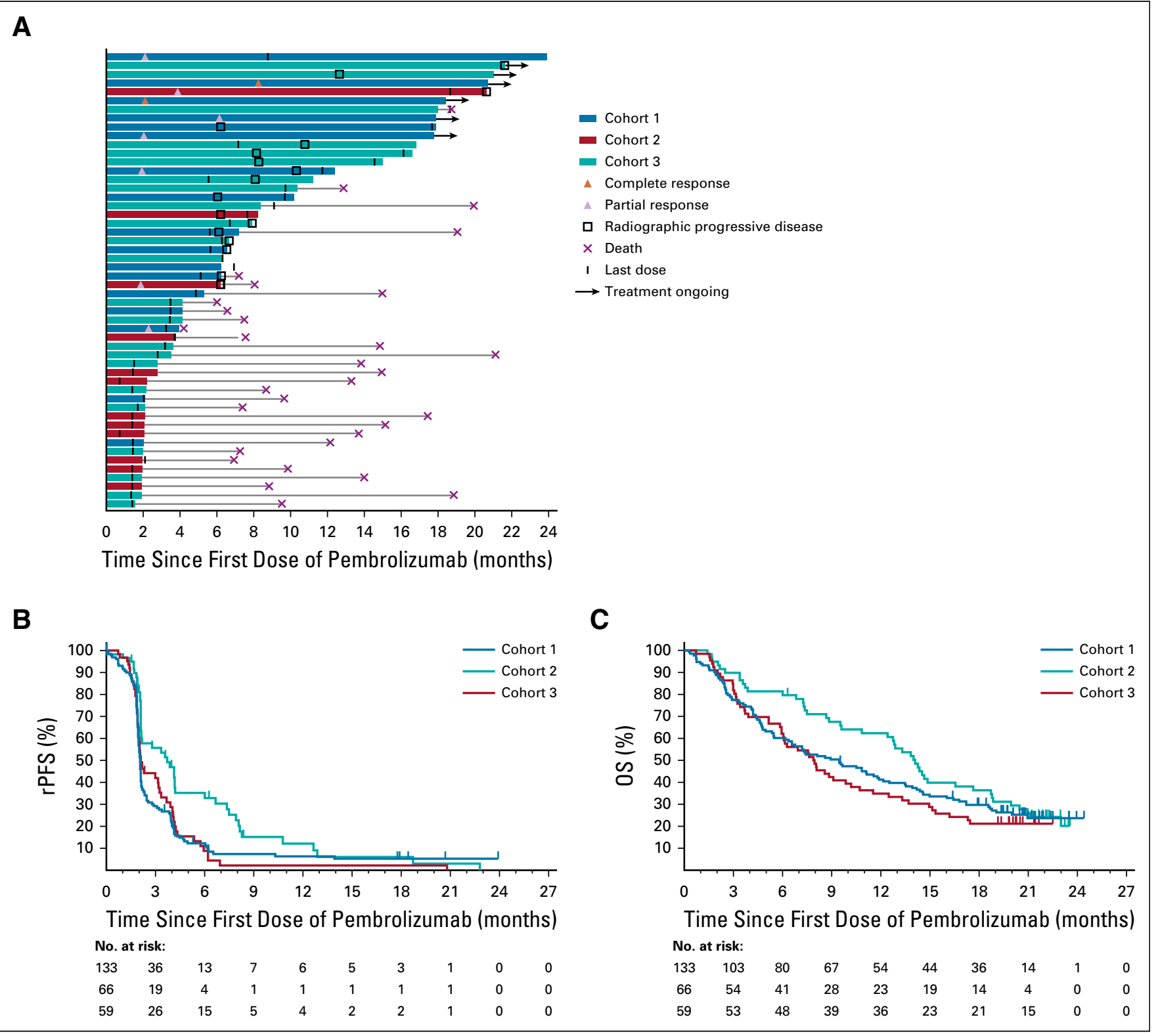

FIG 3. Antitumor activity. (A) Swimmer plot of patients with confirmed response as assessed by Prostate Cancer Working Group 3 (PCWG3)-modified RECIST v1.1 per central review in cohorts $1(n=7)$ and $2(n=2)$ or with stable disease or noncomplete response/nonprogressive disease of 6 or more months duration in cohorts $1(n=10), 2(n=10)$, and $3(n=23)$. Patients who died after month 6 without evidence of disease progression before death were considered to have stable disease for 6 months or longer. The end of the bar indicates the time to the last imaging assessment. (B) Kaplan-Meier estimates of radiographic progression-free survival (rPFS) as assessed by PCWG3-modified RECIST v1.1 per central review in cohorts $1(n=133), 2(n=66)$, and $3(n=$ 59). (C) Kaplan-Meier estimates of overall survival (OS) in cohorts 1, 2, and 3.

patients with previously treated $\mathrm{mCRPC}$, which highlights the importance of identifying predictive biomarkers. ${ }^{42}$ Exploratory biomarker analysis did not seem to identify a clear relationship between response to pembrolizumab and mutations in DDR genes as determined by whole-exome sequencing. The contemporaneous presence of mismatch repair defects could not be excluded, and orthogonal assay testing beyond next-generation sequencing would be necessary to determine functional loss. Although there was no protocol-specified hypothesis testing, outcomes were similar in patients with PD-L1-positive and -negative disease using CPS $\geq 1$ to define positivity. Biomarker analyses were limited by the low number of responses and the availability of whole-exome sequencing results from only a fraction of the population. Ongoing and future biomarker studies from KEYNOTE-199, including exploration of different CPS cut points, gene expression profiles, and tumor mutational burden, will aim to uncover molecular markers of response to single-agent pembrolizumab. ${ }^{42}$ Because the mSINGS assay may provide false negatives, additional assessment of microsatellite instability is also ongoing. ${ }^{43}$ Prospective trials would be required to assess 
TABLE 3. Adverse Events Graded According to Common Terminology Criteria for Adverse Events Version 4.0 in the Combined Population of Cohorts 1,2 , and $3(\mathrm{~N}=258)$

\begin{tabular}{|c|c|c|}
\hline Treatment-related that occu & & \\
\hline Fatigue & $39(15)$ & $3(1)$ \\
\hline Diarrhea & $28(11)$ & $2(<1)$ \\
\hline Decreased appetite & $26(10)$ & $1(<1)$ \\
\hline Nausea & $24(9)$ & 0 \\
\hline Pruritus & $16(6)$ & $1(<1)$ \\
\hline Asthenia & $15(6)$ & 0 \\
\hline AST increased & $11(4)$ & $1(<1)$ \\
\hline Anemia & $10(4)$ & $2(<1)$ \\
\hline Vomiting & $10(4)$ & 0 \\
\hline Constipation & $9(3)$ & 0 \\
\hline ALT increased & $9(3)$ & $2(<1)$ \\
\hline Dyspnea & $9(3)$ & 0 \\
\hline Weight decreased & 7 (3) & 0 \\
\hline Arthralgia & $7(3)$ & 0 \\
\hline Chills & $6(2)$ & 0 \\
\hline Pyrexia & $6(2)$ & 0 \\
\hline Myalgia & $6(2)$ & 0 \\
\hline Rash & $6(2)$ & 0 \\
\hline Immune-mediated and infu & & \\
\hline Colitis & $6(2)$ & $3(1)$ \\
\hline Hyperthyroidism & $6(2)$ & 0 \\
\hline Hypothyroidism & $6(2)$ & $1(<1)$ \\
\hline Pneumonitis & $6(2)$ & $1(<1)$ \\
\hline Severe skin reactions & $6(2)$ & $4(2)$ \\
\hline Infusion reactions & $5(2)$ & $1(<1)$ \\
\hline Hypophysitis & $3(1)$ & $2(<1)$ \\
\hline Adrenal insufficiency & $2(<1)$ & 0 \\
\hline Hepatitis & $1(<1)$ & $1(<1)$ \\
\hline Myositis & $1(<1)$ & 0 \\
\hline Pancreatitis & $1(<1)$ & $1(<1)$ \\
\hline Thyroiditis & $1(<1)$ & 0 \\
\hline Type 1 diabetes mellitus & $1(<1)$ & $1(<1)$ \\
\hline
\end{tabular}

NOTE. Data presented as No. (\%).

*The only grade 5 treatment-related adverse events were pneumonitis and sepsis in one patient each.

†Immune-mediated adverse events and infusion reactions were adverse events of interest based on an expected immunologic mechanism of action. The events were based on a list of terms specified by the sponsor and considered regardless of attribution to treatment or immune relatedness by the investigator. In addition to the preferred terms listed, related terms were also included.

the relationship between DNA repair defects of interest and antitumor activity.

These data add to the growing body of evidence that suggests that, despite its more immunosuppressive microenvironment, a small number of select patients with mCRPC may benefit from pembrolizumab. ${ }^{31,32}$ Data from KEYNOTE-199 cohorts 4 and 5 are pending and will help determine whether pembrolizumab monotherapy has activity in patients with RECIST-measurable (cohort 4) and bone-predominant (cohort 5) chemotherapy-naive mCRPC receiving ongoing enzalutamide treatment. Initial evidence of pembrolizumab monotherapy activity has led to several studies of PD-1 inhibitor-based combination regimens in mCRPC. Data from the multicohort, phase Ib/II KEYNOTE-365 
study demonstrated promising antitumor activity and manageable safety for combinations of pembrolizumab with olaparib, ${ }^{44}$ docetaxel, ${ }^{45}$ and enzalutamide. ${ }^{46}$ Data from the final KEYNOTE-365 cohort of pembrolizumab plus abiraterone and prednisone are pending (ClinicalTrials.gov identifier: NCT02861573). Beyond pembrolizumab and as suggested by an earlier, smaller study, ${ }^{47}$ the phase II CheckMate 650 study showed promising antitumor activity for the combination of nivolumab and ipilimumab in men with mCRPC, although toxicity was a concern and there was a suggestion that prior taxane therapy was associated with lower ORR. ${ }^{48}$ Several phase III studies of pembrolizumab in mCRPC that evaluate response on the basis of PCWG3modified RECIST are ongoing.

\section{AFFILIATIONS}

1Johns Hopkins Sidney Kimmel Comprehensive Cancer Center, Baltimore, MD

${ }^{2}$ Catalan Cancer Institute, Bellvitge Biomedical Research Institute, Centro de Investigación Biomédica en Red de Cáncer, Hospitalet de Llobregat, Barcelona, Spain

${ }^{3}$ Institut Bergonié, Bordeaux, France

${ }^{4}$ Royal Brisbane and Women's Hospital, Herston, QLD, Australia

${ }^{5}$ University of Queensland, St Lucia, QLD, Australia

${ }^{6}$ East Tallinn Central Hospital, Tallinn, Estonia

${ }^{7}$ Case Comprehensive Cancer Center at University Hospitals Seidman Cancer Center, Cleveland, $\mathrm{OH}$

${ }^{8}$ Karmanos Cancer Institute, Wayne State University, Detroit, MI

${ }^{9}$ Chaim Sheba Medical Center, Tel Hashomer, Israel

${ }^{10}$ Başkent University Hospital Adana, Adana, Turkey

${ }^{11}$ Docrates Cancer Center, Helsinki, Finland

${ }^{12}$ Erasmus MC Cancer Institute, Rotterdam, the Netherlands

${ }^{13}$ Karolinska Institutet, Stockholm, Sweden

${ }^{14}$ Cantonal Hospital St Gallen, University of Bern, Bern, Switzerland

${ }^{15}$ Fondazione Istituto Nazionale Tumori, Milan, Italy

${ }^{16}$ Chiba Cancer Center, Chiba, Japan

${ }^{17}$ Kitasato University, Kanagawa, Japan

${ }^{18}$ Sungkyunkwan University, Samsung Medical Center, Seoul, South Korea

${ }^{19}$ Studienpraxis Urologie, Nürtingen, Germany

${ }^{20}$ New York Presbyterian/Columbia University Medical Center, New York, NY

${ }^{21}$ MSD China, Beijing, China

${ }^{22}$ Merck \& Co, Kenilworth, NJ

${ }^{23}$ The Institute of Cancer Research and Royal Marsden Hospital, London, United Kingdom

\section{CORRESPONDING AUTHOR}

Johann Sebastian de Bono, MD, PhD, Regius Professor of Cancer Research, The Institute of Cancer Research and Royal Marsden Hospital, Sycamore House, Downs Rd, Sutton, London, United Kingdom SM2 5PT; e-mail: johann.de-bono@icr.ac.uk.

\section{PRIOR PRESENTATION}

Presented at the 2018 American Society of Clinical Oncology Annual Meeting, Chicago, IL, June 1-5, 2018; and at the 2019 Genitourinary Cancers Symposium, San Francisco, CA, February 14-16, 2019.
This study has several limitations, including the lack of randomization, lack of a control arm, and relatively short follow-up. Because of the inherent biology of metastatic prostate cancer with its tropism to bone, only a proportion of patients provided biopsy samples that could be subjected to whole-exome DNA sequencing. Therefore, the ability to fully characterize the genetic landscape and identify molecular markers of response to pembrolizumab was limited.

In conclusion, pembrolizumab monotherapy shows antitumor activity and disease control with an acceptable safety profile in subsets of patients with RECIST-measurable or bone-predominant mCRPC previously treated with docetaxel and targeted endocrine therapy. Responses seem to be durable, and the observed OS benefit is encouraging.

\section{SUPPORT}

Funded by Merck Sharp \& Dohme, a subsidiary of Merck \& Co (Kenilworth, NJ).

\section{CLINICAL TRIAL INFORMATION \\ NCT02787005}

\section{AUTHORS' DISCLOSURES OF POTENTIAL CONFLICTS OF INTEREST AND DATA AVAILABILITY STATEMENT}

Disclosures provided by the authors and data availability statement (if applicable) are available with this article at DOI https://doi.org/10.1200/ JC0.19.01638.

\section{AUTHOR CONTRIBUTIONS}

Conception and design: Emmanuel S. Antonarakis, Christopher J. Hoimes, Ronald de Wit, Satoshi Fukasawa, Ping Qiu, Christian Poehlein, Johann Sebastian de Bono

Administrative support: Emmanuel S. Antonarakis, Jeffrey Goh, Satoshi Fukasawa, Christian Poehlein

Provision of study material or patients: Emmanuel S. Antonarakis, Josep M. Piulats, Marine Gross-Goupil, Jeffrey Goh, Kristiina Ojamaa, Christopher J. Hoimes, Ulka Vaishampayan, Ranaan Berger, Ahmet Sezer, Tuomo Alanko, Ronald de Wit, Chunde Li, Aurelius Omlin, Giuseppe Procopio, Satoshi Fukasawa, Ken-ichi Tabata, Se Hoon Park, Susan Feyerabend, Charles G. Drake, Johann Sebastian de Bono

Collection and assembly of data: All authors

Data analysis and interpretation: Emmanuel S. Antonarakis, Josep M. Piulats, Christopher J. Hoimes, Ulka Vaishampayan, Ronald de Wit, Chunde Li, Aurelius Omlin, Satoshi Fukasawa, Ken-ichi Tabata, Se Hoon Park, Charles G. Drake, Haiyan Wu, Ping Qiu, Jeri Kim, Christian Poehlein, Johann Sebastian de Bono

Manuscript writing: All authors

Final approval of manuscript: All authors

Accountable for all aspects of the work: All authors

\section{ACKNOWLEDGMENT}

The authors thank the patients and their families and caregivers for participating in this trial, along with all investigators and site personnel. The authors also thank the following employees of Merck Sharp \& Dohme, a subsidiary of Merck \& Co, (Kenilworth, NJ): Scot Ebbinghaus for critical review of the manuscript draft, and Andrew Albright, Razvan Cristescu, Xinwei Sher, and Jialin Xu for assistance in generating and analyzing biomarker data. Medical writing and editorial assistance were provided by Melanie Leiby, an employee of Merck Sharp \& Dohme. 


\section{REFERENCES}

1. Shiao SL, Chu GC, Chung LW: Regulation of prostate cancer progression by the tumor microenvironment. Cancer Lett 380:340-348, 2016

2. Gravis G, Fizazi K, Joly F, et al: Androgen-deprivation therapy alone or with docetaxel in non-castrate metastatic prostate cancer (GETUG-AFU 15): A randomised, open-label, phase 3 trial. Lancet Oncol 14:149-158, 2013

3. James ND, Sydes MR, Clarke NW, et al: Addition of docetaxel, zoledronic acid, or both to first-line long-term hormone therapy in prostate cancer (STAMPEDE): Survival results from an adaptive, multiarm, multistage, platform randomised controlled trial. Lancet 387:1163-1177, 2016

4. Sweeney CJ, Chen YH, Carducci M, et al: Chemohormonal therapy in metastatic hormone-sensitive prostate cancer. N Engl J Med 373:737-746, 2015

5. James ND, de Bono JS, Spears MR, et al: Abiraterone for prostate cancer not previously treated with hormone therapy. N Engl J Med 377:338-351, 2017

6. Fizazi K, Tran N, Fein L, et al: Abiraterone plus prednisone in metastatic, castration-sensitive prostate cancer. N Engl J Med 377:352-360, 2017

7. National Comprehensive Cancer Network: NCCN Clinical Practice Guidelines in Oncology: Prostate cancer, version 3.2018. https://www.nccn.org/ professionals/physician_gls/pdf/prostate.pdf

8. Petrylak DP, Tangen $\mathrm{CM}$, Hussain $\mathrm{MH}$, et al: Docetaxel and estramustine compared with mitoxantrone and prednisone for advanced refractory prostate cancer. N Engl J Med 351:1513-1520, 2004

9. Berthold DR, Pond GR, Soban F, et al: Docetaxel plus prednisone or mitoxantrone plus prednisone for advanced prostate cancer: Updated survival in the TAX 327 study. J Clin Oncol 26:242-245, 2008

10. de Bono JS, Oudard S, Ozguroglu M, et al: Prednisone plus cabazitaxel or mitoxantrone for metastatic castration-resistant prostate cancer progressing after docetaxel treatment: A randomised open-label trial. Lancet 376:1147-1154, 2010

11. Fizazi K, Scher HI, Molina A, et al: Abiraterone acetate for treatment of metastatic castration-resistant prostate cancer: Final overall survival analysis of the COUAA-301 randomised, double-blind, placebo-controlled phase 3 study. Lancet Oncol 13:983-992, 2012

12. Ryan CJ, Smith MR, Fizazi K, et al: Abiraterone acetate plus prednisone versus placebo plus prednisone in chemotherapy-naive men with metastatic castrationresistant prostate cancer (COU-AA-302): Final overall survival analysis of a randomised, double-blind, placebo-controlled phase 3 study. Lancet Oncol 16: 152-160, 2015

13. Scher HI, Fizazi K, Saad F, et al: Increased survival with enzalutamide in prostate cancer after chemotherapy. N Engl J Med 367:1187-1197, 2012

14. Beer TM, Armstrong AJ, Rathkopf DE, et al: Enzalutamide in metastatic prostate cancer before chemotherapy. N Engl J Med 371:424-433, 2014

15. Kantoff PW, Higano CS, Shore ND, et al: Sipuleucel-T immunotherapy for castration-resistant prostate cancer. N Engl J Med 363:411-422, 2010

16. Parker C, Nilsson S, Heinrich D, et al: Alpha emitter radium-223 and survival in metastatic prostate cancer. N Engl J Med 369:213-223, 2013

17. Bellmunt J, de Wit R, Vaughn DJ, et al: Pembrolizumab as second-line therapy for advanced urothelial carcinoma. N Engl J Med 376:1015-1026, 2017

18. Balar AV, Castellano D, O'Donnell PH, et al: First-line pembrolizumab in cisplatin-ineligible patients with locally advanced and unresectable or metastatic urothelial cancer (KEYNOTE-052): a multicentre, single-arm, phase 2 study. Lancet Oncol 18:1483-1492, 2017

19. Sharma P, Retz M, Siefker-Radtke A, et al: Nivolumab in metastatic urothelial carcinoma after platinum therapy (CheckMate 275): A multicentre, single-arm, phase 2 trial. Lancet Oncol 18:312-322, 2017

20. Powles T, Durán I, van der Heijden MS, et al: Atezolizumab versus chemotherapy in patients with platinum-treated locally advanced or metastatic urothelial carcinoma (IMvigor211): A multicentre, open-label, phase 3 randomised controlled trial. Lancet 391:748-757, 2018

21. Balar AV, Galsky MD, Rosenberg JE, et al: Atezolizumab as first-line treatment in cisplatin-ineligible patients with locally advanced and metastatic urothelial carcinoma: A single-arm, multicentre, phase 2 trial. Lancet 389:67-76, 2017

22. Powles T, O'Donnell PH, Massard C, et al: Efficacy and safety of durvalumab in locally advanced or metastatic urothelial carcinoma: Updated results from a phase 1/2 open-label study. JAMA Oncol 3:e172411, 2017

23. Patel MR, Ellerton J, Infante JR, et al: Avelumab in metastatic urothelial carcinoma after platinum failure (JAVELIN Solid Tumor): Pooled results from two expansion cohorts of an open-label, phase 1 trial. Lancet Oncol 19:51-64, 2018

24. Atkins MB, Plimack ER, Puzanov I, et al: Axitinib in combination with pembrolizumab in patients with advanced renal cell cancer: A non-randomised, openlabel, dose-finding, and dose-expansion phase 1b trial. Lancet Oncol 19:405-415, 2018

25. McDermott DF, Sosman JA, Sznol M, et al: Atezolizumab, an anti-programmed death-ligand 1 antibody, in metastatic renal cell carcinoma: Long-term safety, clinical activity, and immune correlates from a phase la study. J Clin Oncol 34:833-842, 2016

26. Ciavarra RP, Holterman DA, Brown RR, et al: Prostate tumor microenvironment alters immune cells and prevents long-term survival in an orthotopic mouse model following flt3-ligand/CD40-ligand immunotherapy. J Immunother 27:13-26, 2004

27. Sfanos KS, Bruno TC, Maris CH, et al: Phenotypic analysis of prostate-infiltrating lymphocytes reveals TH17 and Treg skewing. Clin Cancer Res 14:3254-3261, 2008

28. Pasero C, Gravis G, Guerin M, et al: Inherent and tumor-driven immune tolerance in the prostate microenvironment impairs natural killer cell antitumor activity. Cancer Res 76:2153-2165, 2016

29. Kwon ED, Drake CG, Scher HI, et al: Ipilimumab versus placebo after radiotherapy in patients with metastatic castration-resistant prostate cancer that had progressed after docetaxel chemotherapy (CA184-043): A multicentre, randomised, double-blind, phase 3 trial. Lancet Oncol 15:700-712, 2014

30. Beer TM, Kwon ED, Drake CG, et al: Randomized, double-blind, phase III trial of ipilimumab versus placebo in asymptomatic or minimally symptomatic patients with metastatic chemotherapy-naive castration-resistant prostate cancer. J Clin Oncol 35:40-47, 2017

31. Hansen AR, Massard C, Ott PA, et al: Pembrolizumab for advanced prostate adenocarcinoma: Findings of the KEYNOTE-028 study. Ann Oncol 29:1807-1813, 2018

32. Graff JN, Alumkal JJ, Drake CG, et al: Early evidence of anti-PD-1 activity in enzalutamide-resistant prostate cancer. Oncotarget 7:52810-52817, 2016

33. Eisenhauer EA, Therasse P, Bogaerts J, et al: New response evaluation criteria in solid tumours: Revised RECIST guideline (version 1.1). Eur J Cancer 45: 228-247, 2009

34. Scher HI, Morris MJ, Stadler WM, et al: Trial design and objectives for castration-resistant prostate cancer: Updated recommendations from the Prostate Cancer Clinical Trials Working Group 3. J Clin Oncol 34:1402-1418, 2016

35. Salipante SJ, Scroggins SM, Hampel HL, et al: Microsatellite instability detection by next generation sequencing. Clin Chem 60:1192-1199, 2014

36. Hamid O, Puzanov I, Dummer R, et al: Final analysis of a randomised trial comparing pembrolizumab versus investigator-choice chemotherapy for ipilimumabrefractory advanced melanoma. Eur J Cancer 86:37-45, 2017

37. Herbst RS, Baas P, Kim DW, et al: Pembrolizumab versus docetaxel for previously treated, PD-L1-positive, advanced non-small-cell lung cancer (KEYNOTE010): A randomised controlled trial. Lancet 387:1540-1550, 2016

38. Cohen EEW, Soulières D, Le Tourneau C, et al: Pembrolizumab versus methotrexate, docetaxel, or cetuximab for recurrent or metastatic head-and-neck squamous cell carcinoma (KEYNOTE-040): A randomised, open-label, phase 3 study. Lancet 393:156-167, 2019 
39. Shitara K, Özgüroğlu M, Bang YJ, et al: Pembrolizumab versus paclitaxel for previously treated, advanced gastric or gastro-oesophageal junction cancer (KEYNOTE-061): A randomised, open-label, controlled, phase 3 trial. Lancet 392:123-133, 2018

40. Macedo F, Ladeira K, Pinho F, et al: Bone metastases: An overview. Oncol Rev 11:321, 2017

41. Hernandez RK, Wade SW, Reich A, et al: Incidence of bone metastases in patients with solid tumors: Analysis of oncology electronic medical records in the United States. BMC Cancer 18:44, 2018

42. Antonarakis ES: A new molecular taxonomy to predict immune checkpoint inhibitor sensitivity in prostate cancer. Oncologist 24:430-432, 2019

43. Rodrigues DN, Rescigno P, Liu D, et al: Immunogenomic analyses associate immunological alterations with mismatch repair defects in prostate cancer. J Clin Invest 128:5185, 2018

44. Yu EY, Massard C, Retz M, etal: KEYNOTE-365 cohort A: Pembrolizumab (pembro) plus olaparib in docetaxel-pretreated patients (pts) with metastatic castrateresistant prostate cancer (mCRPC). J Clin Oncol 37, 2019 (suppl 7S; abstr 145)

45. Massard C, Retz M, Hammerer P, et al: KEYNOTE-365 cohort B: Pembrolizumab (pembro) plus docetaxel and prednisone in abiraterone (abi) or enzalutamide (enza)-pretreated patients (pts) with metastatic castrate resistant prostate cancer (mCRPC). J Clin Oncol 37, 2019 (supple 7S; abstr 170)

46. Fong PCC, Retz M, Drakaki A, et al: KEYNOTE-365 cohort C: Pembrolizumab (pembro) plus enzalutamide (enza) in abiraterone (abi)-pretreated patients (pts) with metastatic castrate resistant prostate cancer (mCRPC). J Clin Oncol 37, 2019 (suppl 7S; abstr 171)

47. Boudadi K, Suzman DL, Anagnostou V, et al: Ipilimumab plus nivolumab and DNA-repair defects in AR-V7-expressing metastatic prostate cancer. Oncotarget 9:28561-28571, 2018

48. Sharma P, Pachynski RK, Narayan V, et al: Initial results from a phase II study of nivolumab (NIVO) plus ipilimumab (IPI) for the treatment of metastatic castration-resistant prostate cancer (mCRPC; CheckMate 650). J Clin Oncol 37, 2019 (suppl 7S; abstr 142)

\section{Participate in the Quality Oncology Practice Initiative (QOPI ${ }^{\circledR}$ ) and Demonstrate Your Commitment to Quality Care}

The Quality Oncology Practice Initiative $\left(\mathrm{QOP}{ }^{\circledR}\right)$ is an oncologist-led, practice-based quality assessment program designed to promote excellence in cancer care by helping practices create a culture of self-examination and improvement. The measures are developed and adapted by oncologists and members of the oncology community to ensure they are the most meaningful to our members. QOPI provides the way for you to reliably assess your care relative to these established measures of quality in oncology. The program is an ASCO member benefit, and there is no participation fee.

Get started at qopi.asco.org 


\section{Pembrolizumab for Treatment-Refractory Metastatic Castration-Resistant Prostate Cancer: Multicohort, Open-Label Phase II KEYNOTE-199 Study}

The following represents disclosure information provided by authors of this manuscript. All relationships are considered compensated unless otherwise noted. Relationships are self-held unless noted. I = Immediate Family Member, Inst = My Institution. Relationships may not relate to the subject matter of this manuscript. For more information about ASCO's conflict of interest policy, please refer to www.asco.org/rwc or ascopubs.org/journal/jco/site/ifc.

Open Payments is a public database containing information reported by companies about payments made to US-licensed physicians (Open Payments).

\section{Emmanuel S. Antonarakis}

Honoraria: Sanofi, Dendreon, Medivation, Janssen Biotech, ESSA, Astellas Pharma, MSD, AstraZeneca, Clovis Oncology

Consulting or Advisory Role: Sanofi, Dendreon, Medivation, Janssen Biotech, ESSA, Astellas Pharma, MSD, AstraZeneca, Clovis Oncology

Research Funding: Janssen Biotech (Inst), Johnson \& Johnson (Inst), Sanofi (Inst), Dendreon (Inst), Aragon Pharmaceuticals (Inst), Exelixis (Inst),

Millennium Pharmaceuticals (Inst), Genentech (Inst), Novartis (Inst), Astellas Pharma (Inst), Tokai Pharmaceuticals (Inst), MSD (Inst), AstraZeneca (Inst),

Clovis Oncology (Inst), Constellation Pharmaceuticals (Inst)

Patents, Royalties, Other Intellectual Property: Coinventor of a biomarker technology licensed to Qiagen

Travel, Accommodations, Expenses: Sanofi, Dendreon, Medivation

Josep M. Piulats

Consulting or Advisory Role: Janssen Oncology, Astellas Pharma, VCN

Biosciences, Clovis Oncology, Genentech, Bristol-Myers Squibb, MSD

Research Funding: Bristol-Myers Squibb, AstraZeneca, Medlmmune, MSD

Pfizer, EMD Serono, Incyte, Janssen Oncology

Travel, Accommodations, Expenses: Janssen Oncology, Roche, Bristol-Myers Squibb

Marine Gross-Goupil

Honoraria: Ipsen, Janssen-Cilag, Sanofi

Consulting or Advisory Role: Bristol-Myers Squibb, MSD, Pfizer, Ipsen, Roche

Research Funding: Pfizer, MSD, Roche, Ipsen, Janssen-Cilag

Travel, Accommodations, Expenses: Amgen, Roche, Ipsen, MSD

Jeffrey Goh

Stock and Other Ownership Interests: Immutep

Research Funding: MSD (Inst)

Consulting or Advisory Role: Tesaro, AstraZeneca

Speakers' Bureau: Novartis, Ipsen, Jansen, Mundipharma

Travel, Accommodations, Expenses: Astellas Pharma, AstraZeneca

Kristiina Ojamaa

Consulting or Advisory Role: AstraZeneca

Research Funding: MSD (Inst)

Christopher J. Hoimes

Honoraria: Seattle Genetics

Consulting or Advisory Role: Foundation Medicine, Bristol-Myers Squibb, Eisai,

Prometheus Laboratories, Seattle Genetics, Genentech, MSD, 2bPrecise

Speakers' Bureau: Bristol-Myers Squibb, Genentech

Research Funding: MSD, MSD (Inst)

Ulka Vaishampayan

Honoraria: Pfizer, Bayer, Sanofi, Bristol-Myers Squibb, Exelixis

Consulting or Advisory Role: Pfizer, Bristol-Myers Squibb, Exelixis, Bayer, EMD

Serono

Speakers' Bureau: Pfizer, Bayer, Bristol-Myers Squibb, Exelixis, Sanofi, Eisai

Research Funding: Astellas Pharma, Exelixis, Pfizer, Bristol-Myers Squibb, MSD (Inst)

Raanan Berger

Stock and Other Ownership Interests: Mitra Biotech, Belong

Honoraria: Mitra Biotech, Bristol-Myers Squibb, Roche, AstraZeneca, Pfizer, MSD Oncology

Consulting or Advisory Role: Mitra Biotech, Belong, MSD, Bristol-Myers Squibb

Research Funding: MSD (Inst)

Speakers' Bureau: Mitra Biotech, Bristol-Myers Squibb, MSD

Travel, Accommodations, Expenses: Mitra Biotech, Bristol-Myers Squibb, MSD, AstraZeneca

Ahmet Sezer

Honoraria: Pfizer (Inst), Roche (Inst), Amgen (Inst)

Speakers' Bureau: Roche, Pfizer, Amgen, Bristol-Myers Squibb

Research Funding: Regeneron (Inst), MSD (Inst), Pfizer (Inst), Novartis (Inst) Merck Serono (Inst), Novartis (Inst)

Travel, Accommodations, Expenses: Amgen, Bristol-Myers Squibb, Roche
Tuomo Alanko

Consulting or Advisory Role: Amgen, Bayer, Baxalta, Shire, Bristol-Myers Squibb, Celgene, Eli Lilly, MSD, Netmedi, Nordic Drugs, Roche

Research Funding: AbbVie (Inst), Bayer (Inst), Boehringer Ingelheim (Inst), Bristol-Myers Squibb (Inst), Eli Lilly (Inst), Incyte (Inst), MSD (Inst), Pfizer (Inst), Roche (Inst)

Travel, Accommodations, Expenses: Baxalta, Shire, Bristol-Myers Squibb, MSD, Pfizer, Roche

Ronald de Wit

Honoraria: Sanofi, MSD

Consulting or Advisory Role: Sanofi, MSD, Genentech, Janssen, Bayer, Clovis Research Funding: Sanofi (Inst), Bayer (Inst), MSD (Inst)

Travel, Accommodations, Expenses: Eli Lilly

Chunde Li

Stock and Other Ownership Interests: Chundsell Medicals

Research Funding: MSD (Inst)

Patents, Royalties, Other Intellectual Property: Royalty agreement with

Chundsell Medicals on the basis of a patent granted in Sweden and pending for grant internationally

\section{Aurelius Omlin}

Consulting or Advisory Role: Astellas Pharma (Inst), Bayer (Inst), Sanofi (Inst), Roche (Inst), Janssen (Inst), MSD (Inst), Molecular Partners (Inst)

Research Funding: Teva (Inst), Janssen (Inst), MSD (Inst)

Travel, Accommodations, Expenses: Sanofi, Bayer, Astellas Pharma, Janssen

Giuseppe Procopio

Consulting or Advisory Role: Bayer, Bristol-Myers Squibb, Janssen, Novartis, Astellas Pharma, Pfizer, Ipsen, MSD

Research Funding: MSD (Inst)

\section{Satoshi Fukasawa}

Honoraria: Ono Pharmaceutical, Bristol-Myers Squibb, MSD, Astellas Pharma, AstraZeneca, Novartis, Pfizer, Janssen, Takeda, Daiichi Sankyo

Consulting or Advisory Role: AstraZeneca

Research Funding: MSD (Inst)

Se Hoon Park

Consulting or Advisory Role: Eli Lilly

Research Funding: MSD (Inst)

Susan Feyerabend

Research Funding: MSD (Inst)

Consulting or Advisory Role: Janssen-Cilag, Astellas Pharma

Charles G. Drake

Stock and Other Ownership Interests: Compugen, Tizona Therapeutics, Harpoon Therapeutics, Kleo Pharmaceuticals

Consulting or Advisory Role: AstraZeneca, Medlmmune, Bristol-Myers Squibb, Compugen, Genentech, Janssen Oncology, Pfizer, Tizona Therapeutics,

Potenza Therapeutics, MSD, Pierre Fabre

Research Funding: Bristol-Myers Squibb (Inst), MSD (Inst)

Patents, Royalties, Other Intellectual Property: Patents licensed to Bristol-

Myers Squibb (Inst), Patents licensed to Potenza Therapeutics (Inst)

Travel, Accommodations, Expenses: Genentech, American Association for

Cancer Research, American Society of Clinical Oncology, MSD, Pfizer

\section{Haiyan Wu}

Employment: MSD

Stock and Other Ownership Interests: MSD (I)

Ping Qiu

Employment: MSD

Stock and Other Ownership Interests: MSD

Jeri Kim

Employment: MSD

Stock and Other Ownership Interests: MSD

Christian Poehlein

Employment: MSD

Leadership: MSD

Stock and Other Ownership Interests: MSD 
Johann Sebastian de Bono

Honoraria: AstraZeneca, Sanofi, Astellas Pharma, Pfizer, Genentech, Janssen Oncology, Menarini Silicon Biosystems, Daiichi Sankyo, Sierra Oncology,

\section{Bioexcel}

Consulting or Advisory Role: AstraZeneca, Sanofi, Genentech, Astellas Pharma, Bayer, Pfizer, Merck Sharp \& Dohme, Merck Serono, Boehringer Ingelheim, Sierra Oncology, Menarini Silicon Biosystems, Celgene, Taiho Pharmaceutical, Daiichi Sankyo, Janssen Oncology, Genmab, GlaxoSmithKline, Orion Pharma, Eisai, BioXCel Therapeutics
Research Funding: AstraZeneca (Inst), Genentech (Inst), Sanofi (Inst), Taiho Pharmaceutical (Inst), Daiichi Sankyo (Inst), Merck Serono (Inst), Astex Pharmaceuticals (Inst), Merck Sharp \& Dohme (Inst), Orion Pharma (Inst), GlaxoSmithKline (Inst), CellCentric (Inst), Celgene (Inst), Sierra Oncology (Inst), Bayer (Inst), MedImmune (Inst), Medivation (Inst)

Patents, Royalties, Other Intellectual Property: Abiraterone rewards to inventors (Inst), PARP inhibitors and DNA repair defects (Inst), Targeting of IL-23 in prostate cancer (Inst), CHK1 inhibitor (Inst)

Travel, Accommodations, Expenses: AstraZeneca, Astellas Pharma, GlaxoSmithKline, Orion Pharma, Sanofi, Genmab, Taiho Pharmaceutical, Qiagen, Vertex

No other potential conflicts of interest were reported. 
Pembrolizumab for Treatment-Refractory Metastatic Castration-Resistant Prostate Cancer: Multicohort, OpenLabel Phase II KEYNOTE-199 Study

Emmanuel S. Antonarakis, Josep M. Piulats, Marine Gross-Goupil, Jeffrey Goh, Kristiina Ojamaa, Christopher J. Hoimes, Ulka Vaishampayan, Ranaan Berger, Ahmet Sezer, Tuomo Alanko, Ronald de Wit, Chunde Li, Aurelius Omlin, Giuseppe Procopio, Satoshi Fukasawa, Ken-ichi Tabata, Se Hoon Park, Susan Feyerabend Charles G. Drake, Haiyan Wu, Ping Qiu, Jeri Kim, Christian Poehlein, Johann Sebastian de Bono

\section{METHODS}

Whole-Exome DNA Sequencing. Whole-exome DNA sequences were aligned to reference human genome GRCh37 (Burrows-Wheeler Aligner), variants were detected using GATK, and somatic singlenucleotide variants were called using MuTect. DNA damage repair genes examined were ATM, ATR, BAP1, BARD1, BLM, BRAP, BRCA1, BRCA2, BRIP1, CDH1, CDK12, CENPQ, CHEK1, CHEK2, EPCAM1, ERCC1, ERCC2, ERCC3, ERCC4, ERCC6, FAM175A, FAM175B, FANCA, FANCC, FANCD2, FANCE, FANCF, FANCG, FANCI, FANCL, GEN1, HDAC2, MLH1, MLH3, MRE11A, MSH2, MSH6, MUTYH, NBN, PALB2, PIF1, PMS2, RAD51, RAD51B, RAD51C, RAD51D, RAD54L, RDM1, TP53, and XRCC2. Pathogenic or likely pathogenic mutations were defined as those that were predicted to result in protein loss-homozygous deletions-or protein truncation-nonsense mutations, frameshift insertions or deletions, and splicing mutations at conserved splice donor/acceptor sites-except in the case of TP53, where known inactivation missense mutations were also coded as pathogenic. Sequence alterations not known to alter the encoded protein were denoted as variants of unknown significance.
Definitions of Study End Points and Censoring Rules for Time-

to-Event End Points. Objective response rate was defined as the percentage of patients with confirmed complete or partial response. Disease control rate was defined as the percentage of patients with best overall response of stable disease for 6 months or longer or confirmed complete or partial response. Radiographic progressionfree survival was defined as the time from the first day of study treatment to disease progression or death from any cause, whichever occurred first; data for patients who were alive and did not experience disease progression were censored at the time of last tumor assessment. Overall survival was defined as the time from the first day of study treatment to death from any cause; data for patients who were alive were censored at the time of last contact. The prostate-specific antigen response rate was defined as the percentage of patients with a $50 \%$ or greater reduction in the prostate-specific antigen level from baseline confirmed on an evaluation performed 3 weeks later or longer. Duration of response was defined as the time from first documented evidence of complete or partial response until progressive disease or death as a result of any cause, whichever occurred first, in patients with confirmed complete or partial response.

Censoring rules for duration of response were as follows:

- Data for responders who were alive and without disease progression or new anticancer therapy were censored at the time of last tumor assessment.

- Data for responders who were alive and without disease progression but who started new anticancer therapy were censored at the time of last tumor assessment before the start of the new anticancer therapy.

- Data for responders who died or had disease progression after two or more missed adequate tumor assessments were censored at the time of last adequate tumor assessment before the two or more missed adequate tumor assessments. 
TABLE A1. Summary of Best Overall Response in Cohorts 1, 2, and 3

Cohort 1 (PD-L1 positive; $\mathrm{n}=133$ )
Cohort 2 (PD-L1 negative; $\mathrm{n}=66)$
Cohort 3 (bone predominant;

$\mathrm{n}=59$ )

\begin{tabular}{|c|c|c|c|}
\hline \multicolumn{4}{|l|}{$\begin{array}{l}\text { Response assessed per RECIST v1.1 by } \\
\text { central radiology review }\end{array}$} \\
\hline Complete response & $2(2)$ & 0 & - \\
\hline Stable disease of any duration & $23(17)$ & $14(21)$ & - \\
\hline$\geq 6$-mo duration & $6(5)$ & $4(6)$ & - \\
\hline$\geq 6$-mo duration & 0 & 0 & $13(22)$ \\
\hline Progressive disease & $79(59)$ & $42(64)$ & $32(54)$ \\
\hline Not evaluable† & $4(3)$ & $1(2)$ & $1(2)$ \\
\hline Not assessableł & $20(15)$ & $7(11)$ & $4(7)$ \\
\hline
\end{tabular}

Response assessed per PCWG3-modified

RECIST $v 1.1$ by central radiology review

\begin{tabular}{lccc}
\hline Complete response & $2(2)$ & 0 & 0 \\
\hline Partial response & $5(4)$ & $2(3)$ & 0 \\
\hline Stable disease of any duration & $29(22)$ & $10(15)$ & 0 \\
\hline$\geq 6$-mo duration & $10(8)$ & 0 & $35(59)$ \\
\hline Non-CR/non-PD* of any duration & 0 & 0 & $23(39)$ \\
\hline Progressive disease & 0 & $30(45)$ & $19(32)$ \\
\hline Not evaluable $\dagger$ & $71(53)$ & $2(3)$ & $1(2)$ \\
\hline Not assessable & $6(5)$ & $7(11)$ & $4(7)$
\end{tabular}

NOTE. Data presented as No. (\%).

Abbreviations: CR, complete response; PCWG3, Prostate Cancer Working Group 3; PD, progressive disease; PD-L1, programmed cell death ligand 1.

*Patients without disappearance of all existing lesions or development of new lesions.

†Patients who had one or more postbaseline imaging assessment, none of which were evaluable for response.

$\ddagger$ Patients who did not undergo postbaseline imaging. 
TABLE A2. Tumor and PSA Response by Presence of Monoallelic and Biallelic Aberrations in BRCA1/2, ATM, or Other HRR Genes as Assessed by Whole-Exome Sequencing in Evaluable Patients in Cohorts 1, 2, and 3 Combined

\begin{tabular}{lccc} 
Variable & $\begin{array}{c}\text { BRCA 1/2 or ATM Aberrant } \\
(\mathbf{n = 1 9 )}\end{array}$ & $\begin{array}{c}\text { Aberrations in Other HRR Genes* } \\
(\mathbf{n}=\mathbf{1 0})\end{array}$ & $\begin{array}{c}\text { No Aberrations in HRR Genes } \\
(\mathbf{n}=124)\end{array}$ \\
\hline RECIST v1.1, central review & & & 0 \\
\hline ORR & $2(11) \dagger$ & 0 & $22(18)$ \\
\hline DCR (any duration) & $4(22)$ & & $2(2)$ \\
\hline Best response & & 0 & $2(2)$ \\
\hline CR & 0 & 0 & $18(15)$ \\
\hline PR & $2(11)$ & $2(20)$ & $7(6)$ \\
\hline SD (any duration) & 0 & $80(65)$ \\
\hline Non-CR/non-PD & $2(11)$ & $5(50)$ & $15(12)$ \\
\hline PD & $1(5)$ & $3(30)$ & $4(3)$
\end{tabular}

NOTE. Data presented as No. (\%).

Abbreviations: CR, complete response; DCR, disease control rate; HRR, homologous recombination repair; NE, not evaluable; ORR, objective response rate; $\mathrm{PD}$, progressive disease; $\mathrm{PR}$, partial response; PSA, prostate-specific antigen; $\mathrm{SD}$, stable disease.

*BARD1, BRIP1, CDK12, CHEK1, CHEK2, FANCL, PALB2, PPP2R2A, RAD51C, RAD51B, RAD51D, and RAD54L.

†One patient each from cohorts 1 and 2 .

TABLE A3. Genomic Analysis of 50 DNA Damage Repair Genes in the Six Responders With Evaluable Whole-Exome Sequencing Data

Patient 1 (cohort 1)

ATM splice site acceptor deletion
(chr11_108121426-108121426_A_)

Patient 2 (cohort 1) Patient 3 (cohort 2)

$\begin{array}{cc}\text { TP53 R273P } & \text { BRCA2 } \\ \text { substitution } & \text { V1176Gfs*8 } \\ & \text { insertion }\end{array}$

$\begin{array}{ll}\text { NBN Q494P None } & \text { None } \\ \text { substitution† } & \text { None }\end{array}$

\begin{tabular}{lr}
\hline BRCA2 A1162V substitution† & TP53 S241F \\
& substitution \\
\hline
\end{tabular}

\begin{tabular}{l}
\hline CDK12 G1461Afs* deletion \\
\hline FANCA substitution† \\
(chr16_89816312-89816312_T_C) \\
\hline FANCD2 R263H substitution† \\
\hline MLH3 T930Qfs*35 deletion \\
\hline RAD54L R511H substitution†
\end{tabular}

NOTE. The following 50 DNA damage repair genes were examined: ATM, ATR, BAP1, BARD1, BLM, BRAP, BRCA1, BRCA2, BRIP1, CDH1, CDK12, CENPQ, CHEK1, CHEK2, EPCAM1, ERCC1, ERCC2, ERCC3, ERCC4, ERCC6, FAM175A, FAM175B, FANCA, FANCC, FANCD2, FANCE, FANCF, FANCG, FANCI, FANCL, GEN1, HDAC2, MLH1, MLH3, MRE11A, MSH2, MSH6, MUTYH, NBN, PALB2, PIF1, PMS2, RAD51, RAD51B, RAD51C, RAD51D, RAD54L, RDM1, TP53, and XRCC2.

†Variants of unknown significance. 